\title{
Optical chemical sensor for solving leak detection problems
}

\author{
Aleksandr Maslennikov ${ }^{1, *}$, Ilya Zubkov ${ }^{2}$, V. Pautov ${ }^{2}$ \\ ${ }^{1}$ Nizhny Novgorod State Technical University n.a. R.E. Alekseev, 603950, Minina str., 24, Nizhny \\ Novgorod, Russia \\ ${ }^{2}$ Dzerzhinsk Polytechnic Institute, Nizhny Novgorod State Technical University n.a. R.E. Alekseev, \\ 606039, Gaidara str., 49, Dzerzhinsk, Russia
}

\begin{abstract}
The main features of the construction of gas analytical leak detection systems are noted, from the point of view of the sensitive element and the test substance (gas) used in the leak tightness control. It is shown that when checking the tightness of functioning systems, the standard leak detection equipment is of little use because it is impossible, in most cases, to use the specific test gases necessary for its operation. The use of solidstate gas analytical sensors for the construction of leak detection systems for operating process equipment is proposed. It is shown that one of the most suitable is the optical chemical sensor because of its low sensitivity to the humidity of the carrier gas, which, in such a process of leak detection, is atmospheric air. The properties of the proposed sensor are investigated, which are most important when used in leak detection systems. These parameters are the dynamic characteristics of the sensor, which are determined depending on the operating temperature and pressure of the sensor. The obtained data showed the prospects of using the investigated sensor and also allowed to outline ways of improving and optimizing its design.
\end{abstract}

\section{Introduction}

Leak detection is an extensive and multi-vector industry of technical knowledge, which is primarily due to the wide variety of control objects and the tightness requirements imposed on them. One of the most important groups of leak detection methods are gas analytical methods, the main advantages of which are selectivity and high sensitivity, which is several orders of magnitude higher than the sensitivity of such leak detection methods as the manometric, bubble, and soap indication method.

At the same time, gas analytical methods have a wide range of threshold sensitivities: from $10^{-6}-10^{-8} \mathrm{~m}^{3} \mathrm{~Pa} / \mathrm{s}$ for probe tests or simplified implementations of classical chamber leak detection schemes using halogen or electron-capture leak detectors to extremely high threshold sensitivity values reaching $10^{-10}-10^{-12} \mathrm{~m}^{3} \mathrm{~Pa} / \mathrm{s}$, in the construction of sophisticated chamber leakage control schemes with the accumulation of leakage and the use of mass

\footnotetext{
* Corresponding author: mavdoc@ rambler.ru
} 
spectrometric leak detectors. The objects of control can be both new products and systems, as well as devices that already operate in accordance with their purpose.

When carrying out classic operations of leak testing, a perfectly defined gas is used as a test substance, which is selectively and highly sensitive determined by the exact same leak detector used in this leak test process. If it is a mass spectrometric leak detector, then helium is used as the test gas, if it is halogen, then, as a rule, Freon (SF6). In the case of output production control, problems do not arise, since the same helium, after carrying out the control process without any difficulties, is removed from the control object. Similarly, other test gases used in the leak tightness control can be removed.

But in the event that the object of control is in working order and for this reason is filled with its technological substance, the use of a specific test-flow test substance becomes impossible. Most clearly, this can be demonstrated by the example of the localization of leaks from process plants, inside which there can be a variety of chemicals.

Therefore, a situation arises where the leakage control can only be carried out using the process substance being present inside the equipment. In this case, a gas analytic leak detector is required that is able to selectively detect leaks of this substance. And since there are many technological substances, the types of leak detectors must be large.

The main element of such a leak detector is a gas analytic cell that determines the presence of a gaseous technological substance flowing into the surrounding space. The basis of the cell, taking into account the variety of possible for the determination of substances, can be solid-state gas-analytical sensors, the principles of which are set forth, for example, in [1]. The sensor in the cell determines the presence of the desired substance in the composition of the carrier gas that enters it.

\section{Problem Statement}

A serious problem encountered in the creation of leak detection devices that detect leaks from process equipment is that the definition of such leaks occurs in unstable, in many respects, conditions. First of all, this refers to the temperature and humidity of the ambient air, which in this scheme is a carrier gas of the flowing technological substance into the gas analytic cell. At the same time, many gas analyzers in general and solid-state sensors in particular are very sensitive to such influences. So in [2] the applicability of sensors on surface acoustic waves (SAW-sensor) is analyzed in detail. Like the sensors based on a volumetric acoustic wave (VAW-sensor), the SAW-sensor is a mass-sensitive sensor with a very high threshold sensitivity to the attached mass estimated at 10-12 gr. Such a small value determines the high gas-analytical sensitivity of such sensors. However, the mass-sensitive principle of action leads to the fact that these sensors are sensitive to various influences, leading to a change in the mass of the sensor's gas-sensitive coating. And first of all, such influence is the humidity of the gas environment being analyzed, or more precisely its uncontrolled fluctuations, which leads to an increase in the noise component of the output signal of the sensor, increasing its threshold sensitivity to the concentration of the target substance.

The paper [3] considers the influence of the humidity of the carrier gas on the final signal of the VAW-sensor (AT - cut, 16.5 MHz). It is shown that the effect is very significant. To overcome this problem, two sequential measurements are suggested. First, using a special filter that absorbs the desired substance (heptyl), and then passing the controlled mixture past the filter directly to the sensor. The difference between these two signals reflects the concentration of heptyl. The obvious drawbacks of this approach are the complication of the gas analysis process, and, what is particularly painful for leak testing, the extension of the measurement cycle. 
VAW-sensors are also used to build multi-sensor systems of the "electronic nose" type $[4,5]$, which can include up to ten or more separate sensors whose signals are superimposed by the system to determine the desired substance. The effect of humidity with a large number of sensors is further complicated, creating large errors and uncertainties in determining the concentrations of the substances of interest.

Therefore, for the efficient construction of both gas analytical and leak detection systems, it is important to use sensors with less sensitivity to fluctuations in humidity. These include, in particular, optical chemical sensors [6,7], which perceive changes in color and/or transparency of the sensitive sensor coating and do not sense mass increments.

A great influence on the output signal of solid-state sensors is provided by other environmental parameters, in particular, temperature and pressure. So in [8] a rather detailed analysis of their influence on the static and dynamics of the operation of cold solid-state gasanalytical sensors was carried out. It was shown that this influence is related to the influence of temperature and pressure on the equilibrium constants and the speed of the reversible chemical reaction flowing on the sensor surface between the sensitive sensor coating and the gaseous substance being determined. In the case of physical sorption processes occurring on the surface of the sensor, the interaction mechanisms naturally change, but the influence of both temperature and pressure is preserved.

From the point of view of sensitivity, their selectivity to the test substance and reduction of interference from fluctuations in the humidity of the controlled environment, sensors using a special highly selective gas sensitive film are more preferable. Next, we study exactly such an optical chemical sensor of ammonia. The use of ammonia is due to the urgency of the search for leaks of this substance and its technological use in research.

\section{Research Questions}

This study is a continuation of the investigation of the optical chemical sensor, which was carried out to determine its gas analytical characteristics, which are of the greatest importance in solving purely gas-analytical tasks in eco-monitoring, industrial ecology, and labor protection. Therefore, the static properties of the sensor, their dependence on temperature, and the effect of humidity on the sensor's operation are discussed in detail in the first article also published in this conference proceedings.

Therefore, in order for the considered gas analytical optical chemical sensor to be successfully used in the development of leak detection systems, it is necessary to additionally determine its following parameters: the speed at both the front and the trailing edges of the dynamic characteristic and the effect of temperature and pressure on these parameters.

As done before, the sensitive polymer coating was a functional polymer D924-St, which is an acridine dye immobilized on polystyrene sulfonate.

Thus, the main purpose of this study is to assess the possibility of using the considered optical chemical ammonia sensor in leak detection systems, to determine the operational parameters under which such use would be most effective, and to determine the main directions for optimizing the construction of an optical chemical sensor for further use in leak detection systems.

\section{Research Methods}

To investigate the sensor, an experimental setup was assembled, the circuit of which is shown in Fig. 1.

The installation, basically, is similar to the installation used at the first stage of the study. In order to more accurately assess the dynamic properties of the sensor, it was upgraded in 
the direction of reducing the volume of the chamber and simplifying its internal configuration, which significantly accelerated its purging. To do this, in particular, the moisture sensor was removed from it, and the heater was placed on the outer surface of the chamber. In addition, a block was set up to set the pressure of the test gas medium inside the chamber.

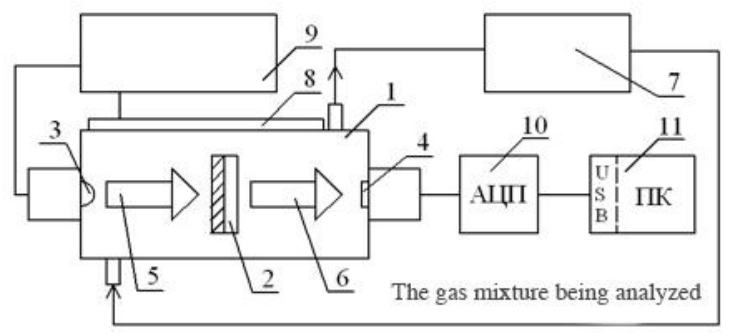

Fig. 1. Experimental installation for the investigation of the optical chemical ammonia sensor: $1-$ camera, 2 - sensor under investigation, 3 - LED, 4 - photodiode, 5 - radiation flux to the sensor, 6 radiation flux after sensor, 7 - pressure sensor in the sensor chamber, 8 - heating element, 9 - control and management unit, 10 - analog-to-digital converter, 11 - personal computer.

To install and maintain the temperature of the camera, a PID controller was used, being equipped with its own temperature sensor. In addition, the chamber is made more massive to reduce fluctuations in its temperature with the imposition of test doses of the ammonia-air mixture. These doses were also chosen for a minimum volume to reduce their effect on the temperature of the medium analyzed by the sensor.

The pressure setting unit allowed to set the absolute pressure in the sensor chamber in the range of 50-250 kPa, after stabilizing the gas environment in the sensor chamber and in the auxiliary chambers connected to it and expanding or compressing the auxiliary chambers. After this, the change in the signal of the optical chemical sensor.

\section{Findings}

The resulting dependencies are presented below in the corresponding figures.

\subsection{Dynamic characteristics}

Figure 2 shows the dependence of the time constant of the optical chemical sensor on the leading and trailing edges of the temperature indicator. At the same time, an ammonia concentration of $-100 \mathrm{mg} / \mathrm{m} 3$ was created in the sensor chamber, which provided a good sensor signal with a low noise level. As can be seen, with increasing temperature, the speed of the sensor increases, and the sensor time constant along the leading edge is about three times smaller than on the trailing edge. This fact is expected, because a rise in temperature increases the rate of both direct and reverse reaction of ammonia with a sensitive sensor coating, reducing the time constants of both fronts.

Since the film of the sensitive coating has a certain thickness, the penetration of ammonia into the interior of the film occurs. It is obvious that the depth of penetration should depend, inter alia, on the number of molecules at the surface of the film, the time of exposure to ammonia, and the diffusion coefficient of ammonia in the film of the sensitive coating. 


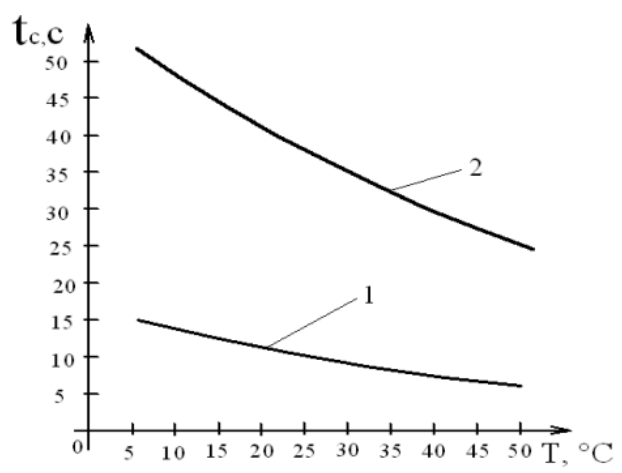

Fig. 2. Dependence of the time constants of the optical chemical sensor on temperature: 1 - on the leading edge, 2 - on the trailing edge.

Therefore, the dependences of the time constants on both fronts of the dynamic characteristic on the affected ammonia concentration were determined. The dependencies are determined for a temperature of $25^{\circ} \mathrm{C}$ and are shown in Figure 3.

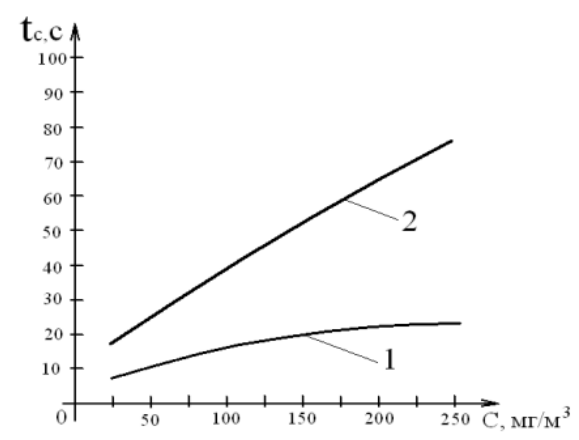

Fig. 3. Dependence of the time constants of the optical chemical sensor on the effect of ammonia concentration: 1 - on the leading edge, 2 - on the trailing edge.

It can be seen that as the effective concentration increases, the time constants increase, although less intensively than the concentration increases. From a practical point of view, this means that it is necessary to protect the sensor from exposure to too high concentrations of ammonia. This can be performed as hardware, blocking the supply of carrier gas to the sensor, for example, from the sensor signal, or organizationally, by monitoring the leak tightness in stages and determining the leaks from large to small, gradually increasing the sensitivity of the monitoring process. This effect, the "poisoning" effect of the sensing element, is not new and is inherent in many highly sensitive gas analytical leaks.

Also, the dependence of the dynamic properties of the optical chemical sensor on the working pressure in the sensor chamber was investigated. The obtained dependences are shown in Fig. 4. 


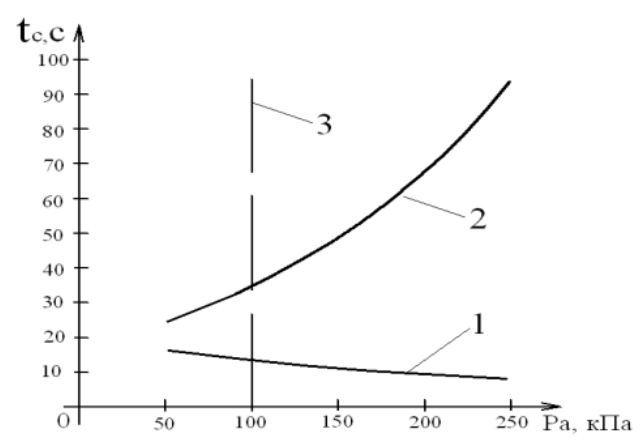

Fig. 4. Dependence of the time constants of the optical chemical sensor on the pressure: 1 - on the leading edge, 2 - on the trailing edge, 3 - atmospheric pressure.

They show that the effect of pressure is multidirectional in relation to the leading and trailing edges of the dynamic characteristic. In particular, it can be seen that increasing the pressure reduces the time constant along the leading edge and increases the time at the rear. This behavior of the time constants is explained by the fact that the increase in pressure increases the rate of direct reaction of the sensitive coating with ammonia and reduces the rate of return.

\subsection{Static characteristics}

Since the increase in pressure varies the rates of direct and reverse reactions differently, it means that the equilibrium constant of this reaction also changes. This, in turn, should change the "output" of the reaction, which, in our case, is equivalent to changing the sensitivity of the sensor. Therefore, the dependence of the sensitivity of the optical chemical sensor on the operating pressure in its chamber was determined, as shown in Fig. 5.

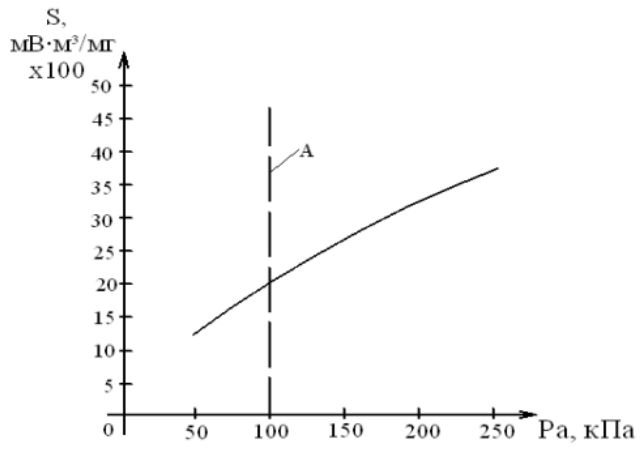

Fig. 5. Dependence of sensitivity of an optical chemical sensor on pressure: A - atmospheric pressure.

It can be seen that increasing the pressure leads to an increase in the sensitivity of the optical chemical sensor, being approximately proportional to the increase in the absolute pressure in the sensor chamber. This dependence, which is quite interesting from the scientific point of view, cannot always be effective from a practical one, especially when leakage is localized, because the technical solutions for introducing the carrier gas into the chamber under excessive pressure can significantly complicate the leak detection equipment. However, in the case of determining the total leakage of the product by the chamber method, 
such a one-time input of the analytical sample may well be carried out, which can significantly increase the sensitivity of the test.

\subsection{Selectivity of the sensor}

Evaluation of the selectivity of the gas analyzer is always partial and incomplete, since it is impossible to determine the reaction of the sensor to all possible substances that can enter its chamber. Therefore, it may be relevant to determine the response of the sensor to the main, most common pollutants of atmospheric air. Sensor reactions to such substances as sulfur dioxide, hydrogen sulfide, nitrogen oxide IV, hydrocarbons (gasoline), alcohols (ethyl and isopropyl), acetone, benzene, toluene were tested. To check the response of the sensor, the concentrations of these substances were used at $100 \mathrm{mg} / \mathrm{m}^{3}$ in air. The reaction of the sensor to their gas-air mixtures was either zero or extremely insignificant, no more than 1 to $3 \%$ of the reaction to a similar concentration of ammonia. This fact, which speaks of the high specificity of the reaction of a sensitive surface with ammonia, is important not only in the leak test, but also in the gas analytic application of the sensor.

\section{Conclusion}

The basic conclusions are made directly in evaluating the obtained dependences of the output signal of the sensor on temperature and pressure. Summarizing the separate conclusions, including taking into account the first article devoted to the gas analytical properties of the optical chemical sensor, we can note the following.

Changes in operating temperature and pressure have a significant effect on the output signal of the sensor. And the temperature has the strongest effect. Increasing the temperature raises the sensitivity of the sensor and simultaneously reduces the values of the time constants along both fronts of its dynamic characteristic. This clearly indicates that it is necessary to increase the operating temperature of the sensor. Such an increase also allows solving the problem of temperature control of the sensor to stabilize its sensitivity, which is especially important for gas analytical applications of an optical chemical sensor, but it will not be superfluous even in its use in leakage. As the maximum operating temperature of the sensor, a temperature in the range of $45-55^{\circ} \mathrm{C}$ can be chosen, since our studies show that the degradation of the sensitive coating at these temperatures is absent. Improving the dynamic properties of sensors with increasing operating temperature is especially important for leak detection devices, but it is also valuable for gas analytical applications. The use of an elevated temperature also improves the protection of the sensor from the high humidity of the carrier gas and allows the sensor, even under the most unfavorable atmospheric conditions, not to leave the low sensitivity range to fluctuations in humidity.

The influence of pressure on the sensor parameters is not so unambiguous. Increasing the pressure leads to an increase in the sensitivity of the sensor but, at the same time, to an increase in the time constant along the trailing edge of the dynamic characteristic. However, the advantage of pressure on the sensor is its rapid implementation. The supply and removal of pressure can be carried out in fractions of a second, which is practically not feasible for temperature effects. Therefore, an occasional decrease in pressure at the trailing edge of the dynamic characteristic of the sensor will significantly accelerate its recovery.

When designing a sensor and a sensor cell, it is important to consider the following. To increase the sensitivity and speed of the sensor itself, it is necessary to develop its design, which has a large number of thin layers of sensitive coating. In addition, it is necessary to minimize the size of the sensor camera, which will also improve both sensitivity and speed in the leak detection of such a sensor. 
In general, it can be argued that the studied sensor, thanks to its sensitive coating, showed very promising characteristics for building on its basis both gas analytical and leak detection systems.

The work was supported by the Ministry of Education and Science of the Russian Federation. Agreement No. 14.577.21.0144 of November 28, 2014. The unique project ID: RFMEFI57714X0144.

\section{References}

1. R. V, Cattrall, Chemical sensors (Scientific World, Moscow, 2000)

2. I. L. Zubkov, A. V. Maslennikov, E. S. Orlov, S. G. Sazhin, Defectoscopiya, 5 (2017)

3. A. A. Grechnikov, A. N. Mogilevsky, Nano- and Microsystem Technology, 2 (2001)

4. T. A. Kuchmenko, E. V. Drozdova, Analytics and Control, 18, 1 (2014)

5. T. A. Kuchmenko, R. P. Lisitskaya, A. A. Shuba, Analytics and Control, 18, 4 (2014)

6. A. A. Egorov, M. A. Egorov, Yu. I. Tsareva, and T. K. Chekhlova, Laser Physics, 17 (2007)

7. E. I. Soborovich, P. A. Bakulin, Sensors and systems, 3 (2000)

8. H. A. Burlaenko, A. B. Maslennikov, S. G. Sazhin, Defectoscopiya, 4 (1996) 